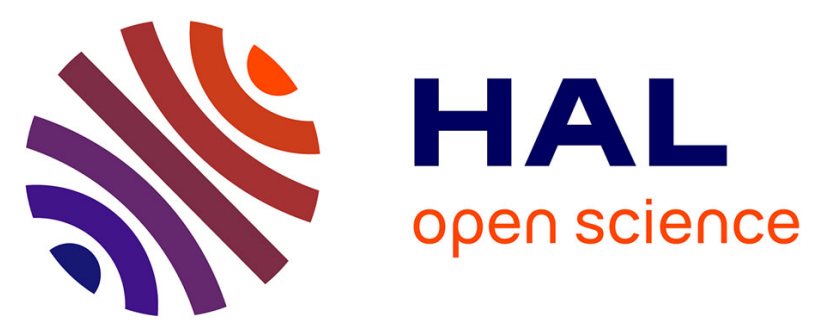

\title{
Self-duality and Digital Topology: Links Between the Morphological Tree of Shapes and Well-Composed Gray-Level Images
}

Thierry Géraud, Edwin Carlinet, Sébastien Crozet

\section{- To cite this version:}

Thierry Géraud, Edwin Carlinet, Sébastien Crozet. Self-duality and Digital Topology: Links Between the Morphological Tree of Shapes and Well-Composed Gray-Level Images. 12th International Symposium on Mathematical Morphology (ISMM, May 2017, Reykjavik, Iceland. pp.573 - 584, 10.1007/9783-319-18720-4_48. hal-01476218

\author{
HAL Id: hal-01476218 \\ https://hal.inria.fr/hal-01476218
}

Submitted on 24 Feb 2017

HAL is a multi-disciplinary open access archive for the deposit and dissemination of scientific research documents, whether they are published or not. The documents may come from teaching and research institutions in France or abroad, or from public or private research centers.
L'archive ouverte pluridisciplinaire HAL, est destinée au dépôt et à la diffusion de documents scientifiques de niveau recherche, publiés ou non, émanant des établissements d'enseignement et de recherche français ou étrangers, des laboratoires publics ou privés. 


\title{
Self-Duality and Digital Topology: Links Between the Morphological Tree of Shapes and Well-Composed Gray-Level Images
}

\author{
Thierry Géraud ${ }^{1}$, Edwin Carlinet $^{12}$, and Sébastien Crozet $^{1}$ \\ 1 EPITA Research and Development Laboratory (LRDE) \\ ${ }^{2}$ Université Paris-Est, LIGM, Équipe A3SI, ESIEE \\ \{firstname.lastname\}@lrde.epita.fr
}

\begin{abstract}
In digital topology, the use of a pair of connectivities is required to avoid topological paradoxes. In mathematical morphology, selfdual operators and methods also rely on such a pair of connectivities. There are several major issues: self-duality is impure, the image graph structure depends on the image values, it impacts the way small objects and texture are processed, and so on. A sub-class of images defined on the cubical grid, well-composed images, has been proposed, where all connectivities are equivalent, thus avoiding many topological problems. In this paper we unveil the link existing between the notion of wellcomposed images and the morphological tree of shapes. We prove that a well-composed image has a well-defined tree of shapes. We also prove that the only self-dual well-composed interpolation of a $2 \mathrm{D}$ image is obtained by the median operator. What follows from our results is that we can have a purely self-dual representation of images, and consequently, purely self-dual operators.
\end{abstract}

Keywords: self-dual operators · tree of shapes · vertex-valued graph · well-composed gray-level images · digital topology.

\section{Introduction}

Having a contrast invariant representation for images is of prime importance in computer vision. Indeed we often have to deal with illumination changes or parts of images being very poorly contrasted [6]5. Some morphological contrast invariant trees have been successfully used for computer vision tasks; see e.g. [23].

In this paper we consider the settings of "digital topology" a-la Rosenfeld and Kong [12]. An image is considered as a vertex-valued graph: the underlying structure is a graph, and an image is a function associating values to vertices [18]. In the following, we only deal with images defined on the $n \mathrm{D}$ cubical grid, so on the square grid for the $2 \mathrm{D}$ case. The objective of this paper is to get a self-dual representation of gray-level images that is free of topological issues. Precisely, we want to guarantee some strong topological properties, to ensure a "pure" self-duality, and to be able consequently to process gray-level images easily and without trouble. Our work has two main motivations. First, we expect to obtain 


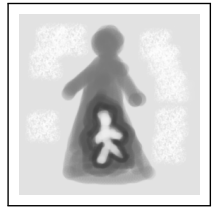

(a)

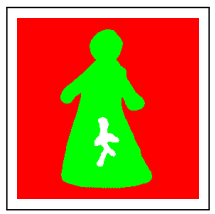

(b)

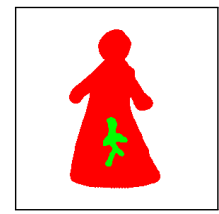

(c)

Fig. 1. In the image (a), the woman can be considered both as foreground (b) or as background (c).

a definition of a discrete tree of shapes that is theoretically sound. Indeed, since this morphological tree-based image representation is self-dual, it is subject to some topological problems. Second, considering a couple of connectivities has a deep impact when dealing with small objects and textures. The cornerstone of our proposal is to handle only one connectedness relationship i.e., a unique topological structure. Put differently, we will consider that an image is one graph structure whose vertices are valued.

The contributions presented in this paper are the following. We show that the tree of shapes is "not purely" self-dual. We prove that, if a gray-level image is well-composed, then its tree of shapes is well defined. We also prove that, under some very reasonable assumptions, the only self-dual well-composed subdivision of a $2 \mathrm{D}$ image is obtained by the median operator. Last we propose a purely self-dual tree of shapes for gray-level images.

First Section 2 recalls the theoretical background of our work. Section 3 explains what we are looking for, and Section 4 gives the solution we propose. Section 5 is dedicated to related works. Last we conclude and give some perspectives in Section 6 .

\section{Theoretical Background}

\subsection{Digital Topology and Self-Duality}

A self-dual operator processes the same way the image contents whatever the contrast (i.e., bright objects over dark background versus dark objects over bright background). That is often desirable when we cannot make an assumption about contrast, and/or when we do not want to make such an assumption because the notion of "object v. background" is not the appropriate one. Actually we often prefer the notion of "subject" (and its related context); this can be explained because the notions of foreground and background are highly contextual.

An illustration of this statement is depicted in Figure 1, where the colors green and red designate respectively the object (foreground) and the background. In the gray-level image, Fig. 1(a), if we take the woman as subject, then we obtain the representation of Fig. 1(b)] and the image outer part is the background. Yet, 
if we take the baby as subject, then we obtain a different interpretation of the image; in Fig. 1(c), the woman is now the background.

Actually, if we take for granted that every part of the image can be a "subject", then we want a unique representation of the image: we do not want a different behavior based on the subject contrast (bright over dark, or the contrary). We thus want to process images in a self-dual way:

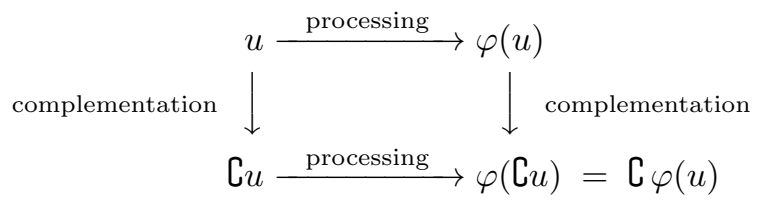

In digital topology in the case of images defined on a regular cubical grid, a "Jordan pair" of connectivities $\left(c_{\alpha}, c_{\beta}\right)$ are required [12: one for the object (foreground), and the other one for the background. Practically, in the 2D case for instance, the choice is $\left(c_{4}, c_{8}\right)$ or $\left(c_{8}, c_{4}\right)$; in $n \mathrm{D}$, it can be $\left(c_{2 n}, c_{3^{n}-1}\right)$ or $\left(c_{3^{n}-1}, c_{2 n}\right)$. The use of the complementation in a self-dual operator thus forces to switch from one connectivity to the other one. That contrasts with the assertion of "processing the same way the image contents whatever the contrast..."; actually we should add "...except for their connectivity".

\subsection{The Morphological Tree of Shapes}

The tree of shapes has been defined in [17, even if its origin comes from [13] in the $2 \mathrm{D}$ case. We just briefly recall here its definition. Given an $n \mathrm{D}$ image $u: \mathbb{Z}^{n} \rightarrow \mathbb{Z}$, the lower level sets are defined as $[u<\lambda]=\{x \in X \mid u(x)<\lambda\}$, and the upper level sets as $[u \geq \lambda]=\{x \in X \mid u(x) \geq \lambda\}$. Considering the connected components of these set: ${ }^{3}$, one can define a couple of dual trees, namely the min-tree $\mathcal{T}_{<}(u)=\{\Gamma \in \mathcal{C C}([u<\lambda])\}_{\lambda}$, and the max-tree $\mathcal{T}_{\geq}(u)=$ $\{\Gamma \in \mathcal{C C}([u \geq \lambda])\}_{\lambda}$. The min-tree and the max-tree verify $\mathcal{T}_{\geq}(\complement u)=\mathcal{T}_{<}(u)$. They are said to be "dual" trees since the operators defined from them are dual.

With the cavity-fill-in operator, denoted by Sat, we have two new sets of components; they are the lower shapes $\mathcal{S}_{<}(u)=\left\{\operatorname{Sat}(\Gamma) ; \Gamma \in \mathcal{T}_{<}(u)\right\}$, and the upper shapes $\mathcal{S}_{>}(u)=\left\{\operatorname{Sat}(\Gamma) ; \Gamma \in \mathcal{T}_{>}(u)\right\}$. The tree of shapes is then defined as:

$$
\mathfrak{S}(u)=\mathcal{S}_{<}(u) \cup \mathcal{S}_{\geq}(u)
$$

and it (almost $\left.{ }^{4}\right)$ features:

$$
\mathfrak{S}(\complement u)=\mathfrak{S}(u) .
$$

Such a tree is called self-dual since many self-dual operators can be derived from this tree 722 .

Last let us recall that a quasi-linear algorithm exists to compute the tree of shapes, that has the property of also working in the $n \mathrm{D}$ case [10]. A parallel version of this algorithm is available 9 .

\footnotetext{
${ }^{3} \mathcal{C C}$ is an operator that takes a set and gives its set of connected components.

${ }^{4}$ We will see in Section 3 that the property $\mathfrak{S}(\complement u)=\mathfrak{S}(u)$ is not strictly correct.
} 


\subsection{Well-Composed Sets and Images}

Let us start by recalling some seminal definitions from the paper [15] by Latecki, Eckhardt, and Rosenfeld.

A 2D set is weakly well-composed if any 8-component of this set is a 4component. A set is well-composed if both this set and its complement are weakly well-composed. A very easy characterization of well-composed sets is based on the notion of "critical configurations"; a set is well-composed if the configurations $\mathbf{\square}$ and $\mathbf{\square}$ do not appear.

The notion of well-composedness has been extended from sets to functions, i.e., gray-level images. A gray-level image $u$ is well-composed if any set $[u \geq \lambda]$ is well-composed. A straightforward characterization of well-composed gray-level images is that every block \begin{tabular}{|l|l|}
\hline$a$ & $d$ \\
\hline$c$ & $b$
\end{tabular} should verify: $\operatorname{intvl}(a, b) \cap \operatorname{intvl}(c, d) \neq \emptyset$, where $\operatorname{intvl}(v, w)=\llbracket \min (v, w), \max (v, w) \rrbracket$.

An image is not a priori well-composed; for instance, the classical "lena" image contains 38039 critical configurations. Two approaches exist to get a wellcomposed image from a primary image: changing its pixel values (yet it can alter the topology of its contents), or getting a well-composed interpolation. Below we give an example of an image (left), which is not well-composed, but whose interpolation (right) is well-composed:

$$
\begin{array}{|l|l|}
\hline 3 & 2 \\
\hline 1 & 8 \\
\hline
\end{array} \quad \begin{array}{|l|l|l|}
\hline 3^{\circ} & 2 & 2 \\
\hline 2 & 2 & 5 \\
\hline 1^{\circ} & 4 & 8^{\circ} \\
\hline
\end{array}
$$

The notion of well-composedness has also been defined for 3D sets and images [14, and it has recently been extended to $n \mathrm{D}$ [2]4. Let us now recap those last results.

Given a point $z \in \mathbb{Z}^{n}$ and a subset $\mathcal{F}=\left\{f_{1}, \ldots, f_{k}\right\}$ of the canonical basis of $\mathbb{Z}^{n}$, with $k \in \llbracket 2, n \rrbracket$, a block $S(z, \mathcal{F})$ associated with $z$ and $\mathcal{F}$ is defined as: $S(z, \mathcal{F})=\left\{z+\sum_{i=1}^{k} \lambda_{i} f_{i} \mid \lambda_{i} \in\{0,1\}, \forall i \in \llbracket 1, k \rrbracket\right\}$. Just remark that a block $S(z, \mathcal{F}) \subset \mathbb{Z}^{n}$ actually belongs to a subspace of dimension $k$. In the following, we will thus say a block $S$ of dimension $k$, meaning that we consider a block $S(z, \mathcal{F})$ such as $\operatorname{card}(\mathcal{F})=k$ and whatever $z$; see Figure 2 for some illustrations.

Given a block $S \subseteq \mathbb{Z}^{n}$ of dimension $k$, and $p, p^{\prime} \in S$, we say that $p$ and $p^{\prime}$ are antagonist in $S$ iff they maximize the distance $L^{1}$ between two points in $S$. Obviously an antagonist to a given point $p \in S$ exists and is unique; it is denoted by $\operatorname{antag}_{S}(p)$. A primary critical configuration of dimension $k$ in $\mathbb{Z}^{n}$ is any set $\left\{p, \operatorname{antag}_{S}(p)\right\}$ with $S$ being a block of dimension $k$. A secondary critical configuration of dimension $k$ in $\mathbb{Z}^{n}$ is any set $S \backslash\left\{p, \operatorname{antag}_{S}(p)\right\}$ with $S$ being a block of dimension $k$.

A set $X \subseteq \mathbb{Z}^{n}$ is well-composed iff, for any block $S$ of dimension $k, X \cap S$ is neither a primary nor a secundary critical configuration. Just notice that the definition of well-composedness is self-dual: any set $X \subseteq \mathbb{Z}^{n}$ is well-composed iff $\mathbb{Z}^{n} \backslash X$ is well-composed. 


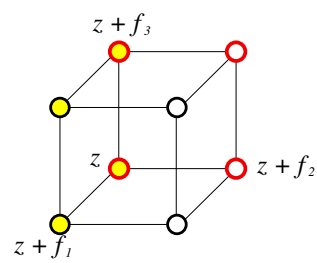

Fig. 2. A block of dimension 3 associated with $z$ contains 8 points of $\mathbb{Z}^{n}$. Two blocks of dimension 2 associated with $z$ are also depicted, each containing 4 points; their points are respectively filled in yellow and contoured in red.

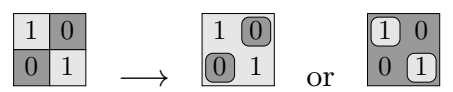

(a) Arbitrary Choice

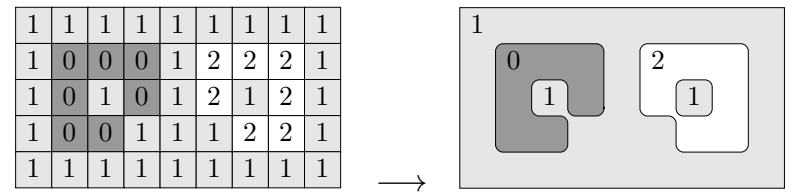

(b) Asymmetry

Fig. 3. Some abnormalities for a "self-dual" representation.

\section{A Quest for Self-Duality}

\subsection{About the Impure Self-Duality}

Let us consider a connectivity $c$ (in $2 \mathrm{D}$ it can be either $c_{4}$ or $c_{8}$ ). Let us denote by $-c$ its "dual" connectivity (the dual of $c_{4}$ is $c_{8}$, and conversely). Let us denote by $\mathcal{R}$ a relation such as $<, \leq,>$, or $\geq$. $\mathcal{R}^{-1}$ denotes the inverse relation (for instance $<^{-1}$ is $>$ ). $\neg \mathcal{R}$ denotes the relation corresponding to its negation (for instance $\neg<$ is $\geq$ since $\neg(a<b) \Leftrightarrow(a \geq b)$. From a set of components:

$$
\mathcal{T}_{(\mathcal{R}, c)}(u)=\left\{\Gamma \in \mathcal{C C}_{c}([u \mathcal{R} \lambda])\right\}_{\lambda},
$$

we can get a set of shapes:

$$
\mathcal{S}_{(\mathcal{R}, c)}(u)=\left\{\operatorname{Sat}_{-c}(\Gamma) ; \Gamma \in \mathcal{T}_{(\mathcal{R}, c)}(u)\right\} .
$$

Note that, from a component $\Gamma$ obtained with the connectivity $c$, the cavity-fill operator relies on the connectivity $-c$ to be topologically sound. Then just remark that the cavities of the components of $\mathcal{T}_{(\mathcal{R}, c)}(u)$ are some shapes belonging to $\mathcal{S}_{(\neg \mathcal{R},-c)}(u)$. So we can define the tree of shapes by:

$$
\mathfrak{S}_{(\mathcal{R}, c)}(u)=\mathcal{S}_{(\mathcal{R}, c)}(u) \cup \mathcal{S}_{(\neg \mathcal{R},-c)}(u) .
$$

Note that, in the discrete case, there is no difference between the sets $\mathcal{T}_{(\mathcal{R}, c)}(u)$ and $\mathcal{T}_{\left(\neg \mathcal{R}^{-1}, c\right)}(u)($ e.g., the same threshold sets are obtained using $<$ or $\leq)$. We 


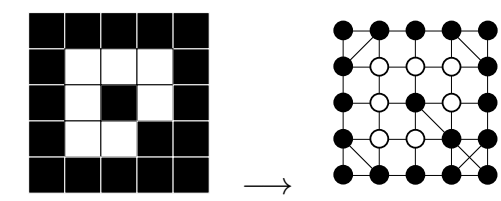

Fig. 4. A set (left) and its corresponding graph (right).

can finally observe that the tree of shapes is not purely self-dual:

$$
\mathfrak{S}_{(\mathcal{R}, c)}(\complement u)=\mathfrak{S}_{\left(\mathcal{R}^{-1}, c\right)}(u)=\mathfrak{S}_{\left(\neg \mathcal{R}^{-1},-c\right)}(u)=\mathfrak{S}_{(\mathcal{R},-c)}(u) .
$$

For instance in $2 \mathrm{D}$, we have: $\mathfrak{S}_{\left(<, c_{4}\right)}(\mathfrak{C} u)=\mathfrak{S}_{\left(<, c_{8}\right)}(u)$.

Now consider a "self-dual" operator depending upon a connectivity pair $\left(c_{\alpha}, c_{\beta}\right)$; we have the following scheme:

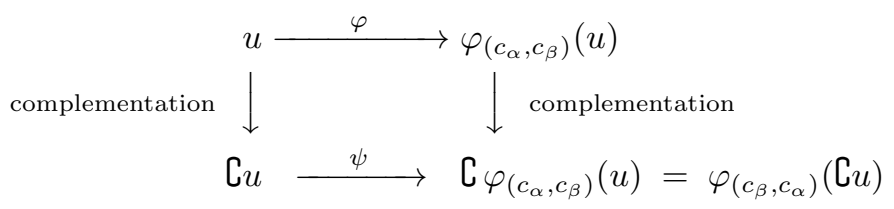

so precisely $\varphi_{\left(c_{\alpha}, c_{\beta}\right)}$ and $\varphi_{\left(c_{\beta}, c_{\alpha}\right)}$ are dual. We cannot formally say that $\varphi$ is self-dual because we do not have $\complement \varphi_{\left(c_{\alpha}, c_{\beta}\right)}=\varphi_{\left(c_{\alpha}, c_{\beta}\right)} \complement$.

In both cases of the tree of shapes and of a self-dual operator, the underlying representation of an image depends upon the arbitrary choic $e^{5}$ of taking either $\left(c_{\alpha}, c_{\beta}\right)$ or $\left(c_{\beta}, c_{\alpha}\right)$; such a choice is illustrated by Fig. 3(a) Consequently it yields an asymmetry in dealing with components, which is illustrated by Fig. 3(b). In those cases, we will say that self-duality is not pure.

\subsection{Images as Vertex-Valued Graphs}

If we consider an image as a vertex-valued graph, the use of a connectivity pair is a priori mandatory to avoid the connectivity paradoxes of digital topology. Figure 4 depicts a binary image (a set), and its corresponding graph, where the connectivities $\left(c_{4}, c_{8}\right)$ have been chosen to represent respectively the foreground (white) and the background (black).

On the other hand, we want a purely self-dual tree, i.e., a tree that strictly verifies $\mathfrak{S}(\complement u)=\mathfrak{S}(u)$. That starts with a first requirement: we shall have the same connectivity relation, say $c$, for both lower and upper shapes. Remind now that the lower (resp. upper) shapes come from the cavity-fill operator applied on some components of the upper (resp. lower) threshold sets. It leads to the conclusion that a unique connectivity shall be used everywhere (precisely to define the components of all threshold sets, and to define the cavity-fill operator

\footnotetext{
${ }^{5}$ Note that the classical workaround to ensure pure self-duality and avoid topological problems using 6-connectivity in $2 \mathrm{D}$ is out of scope, since it required a choice between 4 possible transforms (shifting either rows or columns, and either odd or even ones).
} 
for both lower and upper components). The definitions of the previous section shall be rewritten as follows. From components we get shapes:

$$
\mathcal{T}_{(\mathcal{R}, c)}(u)=\left\{\Gamma \in \mathcal{C C}_{c}([u \mathcal{R} \lambda])\right\}_{\lambda} \longrightarrow \mathcal{S}_{(\mathcal{R}, c)}(u)=\left\{\operatorname{Sat}_{c}(\Gamma) ; \Gamma \in \mathcal{T}_{(\mathcal{R}, c)}(u)\right\}
$$

and we take the union of lower and upper shapes:

$$
\mathfrak{S}_{(\mathcal{R}, c)}(u)=\mathcal{S}_{(\mathcal{R}, c)}(u) \cup \mathcal{S}_{(\mathcal{R}, c)}(\complement u) .
$$

Finally, the components of $\mathfrak{S}_{(\mathcal{R}, c)}(u)$, endowed with inclusion, do not form a tree, but a poset.

As a conclusion, if we consider that an image is a function, we cannot separate the structure of the definition domain from the values of the image. Put differently, it means that the graph structure depends on the image values. That is a major issue, since it is a very strong restriction.

\subsection{Rationale}

The rationale behind our proposal is the following. Given any gray-level $n \mathrm{D}$ image $u,\left(\mathfrak{S}_{(\mathcal{R}, c)}(u), \subset\right)$ forms a poset. Taking $c=c_{\alpha}$ or $c=c_{\beta}$ is equivalent when an image is well-composed. We can define a self-dual interpolation $\mathfrak{I}(u)$ of $u$ that is a well-composed image. So we can expect $\mathfrak{S}_{(\mathcal{R}, c)}(\mathfrak{I}(u))$ to be a purely self-dual tree of shapes. To remain consistent with both digital topology and mathematical morphology, we will impose some reasonable constraints on $\mathfrak{I}$.

\section{Getting a Purely Self-Dual Tree of Shapes}

\subsection{Well-Composed $\Rightarrow$ Tree of Shapes}

Actually there is a link between the notion of well-composed images and the definition of the tree of shapes:

Theorem. If a gray-level $n \mathrm{D}$ image $u$ is well-composed, then the components of $\mathfrak{S}_{\left(\mathcal{R}, c_{2 n}\right)}(u)$ form a (purely) self-dual tree of shapes.

Let us first remark that it is an implication, not an equivalence (being wellcomposed is a sufficient condition to get a tree of shapes; it is not a necessary one). Indeed, with:

$$
u=\begin{array}{|l|l|l|l|}
\hline 1 & 1 & 1 & 1 \\
\hline 1 & 0 & 2 & 1 \\
\hline 1 & 2 & 0 & 1 \\
\hline 1 & 1 & 1 & 1 \\
\hline
\end{array}
$$

$\mathfrak{S}_{\left(<, c_{2 n}\right)}(u)$ is a tree, while $u$ is not well-composed.

Proof. A strong recent result, presented in [4], is the following: "if a set $X \subseteq \mathbb{Z}^{n}$ is well-composed, then its $2 n$-components are identical to its $\left(3^{n}-1\right)$-components". That implies that:

$$
\mathfrak{S}_{\left(\mathcal{R}, c_{2 n}\right)}(\mathfrak{\complement} u)=\mathfrak{S}_{\left(\mathcal{R}, c_{3^{n}-1}\right)}(u)=\mathfrak{S}_{\left(\mathcal{R}, c_{2 n}\right)}(u),
$$


which means that $u$ and $\complement u$ have the same set of shapes. Consequently this set is (said) self-dual, since purely self-dual operators can be defined from it.

Let us show now that the elements of $\mathfrak{S}_{\left(\mathcal{R}, c_{2 n}\right)}(u)$ form a tree. The proof that the shapes obtained with $\left(c_{2 n}, c_{3^{n}-1}\right)$ form a tree can be found in [18]. Since the connectivities $c_{2 n}$ and $c_{3^{n}-1}$ are equivalent for a well-composed image, this proof applies to our case.

\subsection{A Self-Dual 2D Morphological Interpolation}

We are now going to study how to interpolate an image $u$ defined on $\mathbb{Z}^{n}$ into an image $\mathfrak{I}(u)$ defined on $(\mathbb{Z} / 2)^{n}$. The requirements over the interpolation $\mathfrak{I}$ are the following ones. 1. It shall commute with the classical geometric reflections and rotations, i.e., $\mathfrak{I} \circ T=T \circ \mathfrak{I}$, with $T$ such a transform. 2. $\mathfrak{I}(u)$ shall be considered as a rasterization equivalent to $u$ (cf. [1621); in particular, it shall not create some new extremum. 3. It shall be self-dual, i.e., $\mathfrak{I}(\complement u)=\complement \mathfrak{I}(u)$. 4. We shall ensure that the shapes are invariant to contrast changes (a classical morphological axiom): $\mathfrak{S}(g(\mathfrak{I}(u)))=\mathfrak{S}(\mathfrak{I}(g(u)))$, where $g$ is a strictly increasing function. 5. Last, the interpolation function, used to set values between the original pixels, shall actually be an operator. Note that we will also use the notation $\mathfrak{I}$ for this operator.

Consider a $3 \times 3$ pixels piece of $\mathfrak{I}(u)$ and a threshold set $X$. We will depict respectively by $\bullet$ an element of $X$, by $\bullet$ an element of $\complement X$, and by $\circ$ an element for which we do not know if it is in $X$ or not. It yields 4 cases (modulo symmetries, rotations, and complementation). Using only the "no new extremum" constraint, we have:

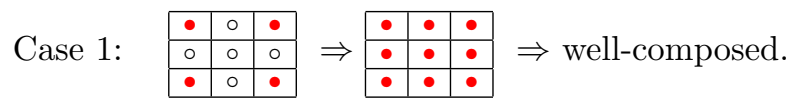

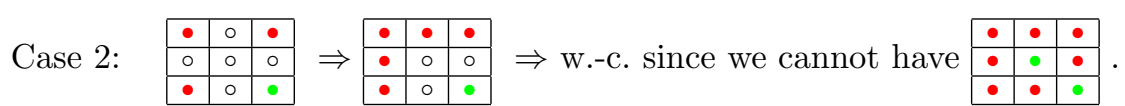

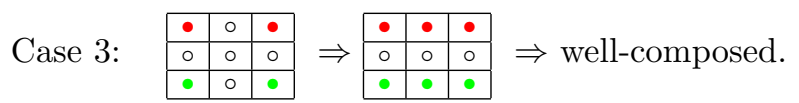

Case 4: \begin{tabular}{|c|c|c|}
\hline$\bullet$ & 0 & $\bullet$ \\
\cline { 2 - 2 } & $\circ$ & $\circ$ \\
\hline & 0 & $\bullet$
\end{tabular}$\Rightarrow$ we have to study this "saddle-point" case.

Let us assume that the pixel values are $a<b<c<d$, and that the interpolation of this piece of image is:

\begin{tabular}{|c|c|c|}
\hline$a$ & $a d$ & $d$ \\
\hline$a c$ & $a b c d$ & $b d$ \\
\hline$c$ & $b c$ & $b$ \\
\hline
\end{tabular}

where we shorten a notation such as $\mathfrak{I}\{v, w\}$ into $v w$, and $\Im\{a, b, c, d\}$ into abcd. Now, just remark that the "no new extremum" and "self-dual" constraints imply that $a<d \Rightarrow a<a d<d$, and we have the similar ordering using $\{a, c\},\{c, b\}$, and $\{b, d\}$. Last, this same constraint also implies that $a c<$ 
$a b c d<b d$ and $a d<a b c d<b c$. We can then deduce the Hasse diagram of the set of values, depicted on the left below:
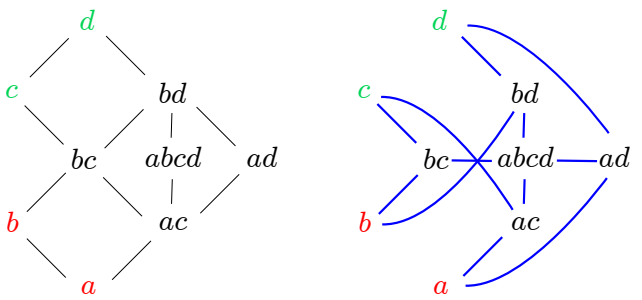

When only drawing between these values the 4-adjacencies of their pixels, we obtain the diagram depicted above on the right side. For instance, the point whose value is $b$ is 4 -adjacent to the points whose values are $b c$ and $b d$. Remark that we have maintain the locations of values of the Hasse diagram, which allows us for keeping reasoning on value ordering.

Assume that the point of value $a c$ is in $X$; so it is depicted in green below. Since we have $a c<a b c d<b d$ the points with values abcd and $b d$ are in $X$, so they are depicted in green. We end up with the image piece, depicted below, which is well-composed (whatever its unknown part):

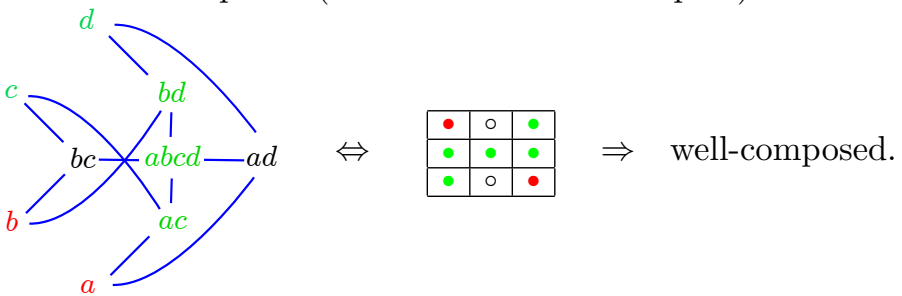

The assumption that the point of value $b d$ is in $\complement X$ is the dual assumption of the previous one (with finally abcd and $a c$ in $\complement X$ ); it also leads to a well-composed image piece.

So there is only one remaining case, $b d \in X$ and $a c \in \complement X$, depicted below. For this image piece to be well-composed, we can see that the points depicted in blue $(\bullet)$, corresponding to the values $a b c d$ and $b c$, have to be in the same set (either $X$ or $\complement X$ ). Finally we shall have $a b c d=b c$ :

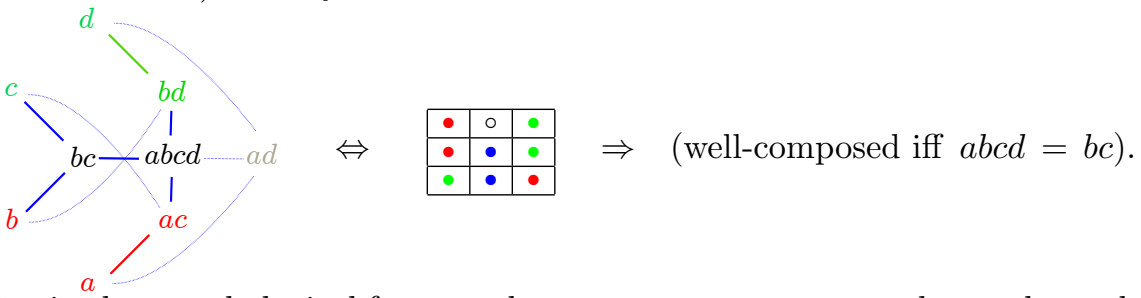

Being in the morphological framework, we want an operator so the result can be rewritten as $\mathrm{op}(\{a, b, c, d\})=\mathrm{op}(\{b, c\})$. It should be true whatever the values, so the operator is $a$ median.

Let us recall that we have assumed that, in the piece of image considered, the pixel values are all different $(a<b<c<d)$. If we have now $a \leq b \leq c \leq d$, we actually get the same conclusions as before. Indeed, just remark that having 
some values equal means that the Hasse diagram is simplified, yet not really modified.

Let us consider a multi-set $S=\left\{z_{1}, \ldots, z_{k}\right\}$ of $k$ integers, with $k$ even, such as $\forall i \in \llbracket 1, k-1 \rrbracket, z_{i} \leq z_{i+1}$. We can define the median operator by $\operatorname{med}(S)=$ $\left(z_{\frac{k}{2}}+z_{\frac{k}{2}+1}\right) / 2$; note that $\operatorname{med}(S) \in \mathbb{Z} / 2$. This operator satisfies the property:

$\forall S_{1}$ and $S_{2}$ such as $S=S_{1} \cup S_{2}, \operatorname{med}(S) \in \operatorname{intvl}\left(\operatorname{med}\left(S_{1}\right), \operatorname{med}\left(S_{2}\right)\right)$,

so med is an interpolation operator that does not create new extremum. Indeed, we have for instance $\operatorname{med}\{a, d\}=(a+d) / 2 \in \operatorname{intvl}(a, d)$, and at the center of an image piece: $\operatorname{med}\{a, b, c, d\} \in \operatorname{intvv}(\operatorname{med}\{a, c\}, \operatorname{med}\{b, d\})$, and $\operatorname{med}\{a, b, c, d\} \in$ $\operatorname{intvl}(\operatorname{med}\{a, d\}, \operatorname{med}\{b, c\})$. In addition, the operator med is self-dual: $\operatorname{med}(S)=$ $-\operatorname{med}\left\{-z_{k}, \ldots,-z_{1}\right\}$.

In $2 \mathrm{D}$, we thus have a med-based interpolation operator, $\mathfrak{I}_{\text {med }}$, that transforms an image $u: \mathbb{Z}^{2} \rightarrow \mathbb{Z}$ into $\mathfrak{I}_{\text {med }}(u):(\mathbb{Z} / 2)^{2} \rightarrow \mathbb{Z} / 2$. Formally, with $B=\left\{-\frac{1}{2}, 0, \frac{1}{2}\right\}^{2}$, and with $B_{z}$ the translation of $B$ by $z$, we have:

$$
\forall z \in(\mathbb{Z} / 2)^{2}, \quad\left[\mathfrak{I}_{\text {med }}(u)\right](z)= \begin{cases}u(z) & \text { if } z \in \mathbb{Z}^{2} \\ \operatorname{med}\left\{u\left(z^{\prime}\right), z^{\prime} \in B_{z} \cap \mathbb{Z}^{2}\right\} & \text { otherwise }\end{cases}
$$

which is a well-composed self-dual $2 \mathrm{D}$ interpolation of $u$ verifying the desired properties and invariances.

In this section, we have actually proven Proposition 25 from [19: "The median interpolation of a function defined on $\mathbb{Z}^{2}$ leads to a self-dual plain map." Last, let us mention that this interpolation, once generalized in $n \mathrm{D}$, does not offer the guaranty to produce well-composed images when $n \geq 3$; a 3D example given in [3] is: $u=\left(\begin{array}{ll|ll}\mathbf{2} & \mathbf{4} & \mathbf{4} & \mathbf{0} \\ \mathbf{4} & \mathbf{0} & \mathbf{0} & \mathbf{2}\end{array}\right) \rightarrow \mathfrak{I}_{\text {med }}(u)=\left(\begin{array}{lll|lll|lll}\mathbf{2} & 3 & \mathbf{4} & 3 & 3 & 2 & \mathbf{4} & 2 & \mathbf{0} \\ 3 & 3 & 2 & 3 & 2 & 1 & 2 & 1 & 1 \\ \mathbf{4} & 2 & \mathbf{0} & 2 & 1 & 1 & \mathbf{0} & 1 & \mathbf{2}\end{array}\right)$, where a $2 \mathrm{D}$ critical configuration is depicted in italics .

\section{Related Works}

In [20], Ray and Acton give a proof that an inclusion tree exists when considering the connectivity pair $\left(c_{8}, c_{8}\right)$. Unfortunately, not having a Jordan pair yields some results that are difficult to understand, since they do not conform to what can be expected in the continuous case. For instance, with the image given at the beginning of Section 4.1. the two central components (containing 2 points each, and with the respective levels 0 and 2) cross using $c_{8}$; it means that their respective contours (level lines) also cross, although these two contours have different levels.

In 18 , the proof that the shapes obtained with $\left(c_{2 n}, c_{3^{n}-1}\right)$ form a tree relies on changing a discrete function $u: \mathbb{Z}^{n} \rightarrow \mathbb{R}$ into an upper semi-continuous function $u_{\max }: \mathbb{R}^{n} \rightarrow \mathbb{R}$. Actually, this latter function can be related to the interpolation $\mathfrak{I}_{\max }(u)$, obtained such as $\mathfrak{I}_{\text {med }}(u)$ while replacing med by max. It is easy to see that $\mathfrak{I}_{\max }(u)$ is a well-composed discrete interpolation of $u$, which is not self-dual. Its dual interpolation, also well-composed, is $\mathfrak{I}_{\min }(u)$. 
In [19, Najman and Géraud have proposed an interval-valued interpolation of $n \mathrm{D}$ images, based on the Khalimsky grid. They have given an alternative definition of well-composed $n \mathrm{D}$ images: the components of the boundaries of all threshold sets are discrete $(n-1)$-manifolds. Yet, this definition is different from the one we use in the present paper (see Section 2.3) based on the notion of critical configurations [2]. To our knowledge, the equivalence (or non-equivalence) between both definitions has not been proven yet.

Several authors have proposed some methods to "repair" a 3D set so that the result is well-composed; a bibliography can be found in [11. In these methods the notions of critical configurations are involved but, unlike us, they do not consider the simple setting of "digital topology".

In [4, Boutry et al. have presented a self-dual $n \mathrm{D}$ interpolation that is wellcomposed, whatever the dimension $n$. The major difference between their result and what we have proposed in Section 4.2 is that they do not impose the interpolation function to be local.

\section{Conclusion}

In this paper, we have shown that we need well-composed images to get (a really pure) self-duality, and we have proven that a median-based interpolation is the solution to get $2 \mathrm{D}$ well-composed images. A major consequence is that an $n \mathrm{D}$ image defined on the cubical grid can really be seen as a function valuing the vertices of a graph (otherwise the graph depends upon the image values). In particular, that means that we can have two different functions (set of values) defined on the same graph, since the domain structure can be truly uncorrelated from the valuation. The drawbacks of our proposal is that we need to subdivide the domain (yet it does not change any operator / method complexity because it is just a multiplicative factor, and RAM is cheap). The perspectives of this work are rather straightforward: finding if we can generalize the properties of well-composed sets and images to any graph (not only cubical grids), and evaluate the advantages of our $2 \mathrm{D}$ proposal versus an interpolation dedicated to 6-connectivity, which is intrinsically topology paradox free.

Acknowledgment. The authors want to thank Laurent W. Najman for his constant support and good advice.

\section{References}

1. Ballester, C., Caselles, V., Monasse, P.: The tree of shapes of an image. ESAIM: Control, Optimisation and Calculus of Variations 9, 1-18 (2003)

2. Boutry, N., Géraud, T., Najman, L.: A generalization of well-composedness to dimension $n$. Communication at Journée du Groupe de Travail de Géometrie Discrète (GT GeoDis, Reims Image 2014) (Nov 2014), in French.

3. Boutry, N., Géraud, T., Najman, L.: On making $n \mathrm{D}$ images well-composed by a self-dual local interpolation. In: Proceedings of the 18th International Conference on Discrete Geometry for Computer Imagery (DGCI). Lecture Notes in Computer Science, vol. 8668, pp. 320-331. Springer, Siena, Italy (September 2014) 
4. Boutry, N., Géraud, T., Najman, L.: How to make $n \mathrm{D}$ images well-composed in a self-dual way. In: Proceedings of ISMM (2015), This volume

5. Cao, F., Lisani, J.L., Morel, J.M., Musé, P., Sur, F.: A Theory of Shape Identification, Lecture Notes in Mathematics, vol. 1948. Springer (2008)

6. Caselles, V., Coll, B., Morel, J.M.: Topographic maps and local contrast changes in natural images. International Journal of Computer Vision 33(1), 5-27 (1999)

7. Caselles, V., Monasse, P.: Grain filters. Journal of Mathematical Imaging and Vision 17(3), 249-270 (2002)

8. Caselles, V., Monasse, P.: Geometric Description of Images as Topographic Maps, Lecture Notes in Mathematics, vol. 1984. Springer-Verlag (2009)

9. Crozet, S., Géraud, T.: A first parallel algorithm to compute the morphological tree of shapes of $n \mathrm{D}$ images. In: Proceedings of the 21st International Conference on Image Processing (ICIP). pp. 2933-2937. Paris, France (2014)

10. Géraud, T., Carlinet, E., Crozet, S., Najman, L.: A quasi-linear algorithm to compute the tree of shapes of $n$-D images. In: Proceedings of the 11th International Symposium on Mathematical Morphology (ISMM). Lecture Notes in Computer Science Series, vol. 7883, pp. 98-110. Springer, Heidelberg (2013)

11. Gonzalez-Diaz, R., Jimenez, M.J., Medrano, B.: 3D well-composed polyhedral complexes. Discrete Applied Mathematics 183, 59-77 (March 2015), special Issue on Discrete Geometry for Computer Imagery

12. Kong, T.Y., Rosenfeld, A.: Digital topology: Introduction and survey. Computer Vision, Graphics, and Image Processing 48(3), 357-393 (December 1989)

13. Kronrod, A.: On functions of two variables. Uspehi Mathematical Sciences 5, 24 134 (1950), in Russian.

14. Latecki, L.: 3D well-composed pictures. Graphical Models and Image Processing 59(3), 164-172 (May 1997)

15. Latecki, L., Eckhardt, U., Rosenfeld, A.: Well-composed sets. Computer Vision and Image Understanding 61(1), 70-83 (January 1995)

16. Latecki, L.J., Conrad, C., Gross, A.: Preserving topology by a digitization process. Journal of Mathematical Imaging and Vision 8(2), 131-159 (March 1998)

17. Monasse, P., Guichard, F.: Fast computation of a contrast-invariant image representation. IEEE Transactions on Image Processing 9(5), 860-872 (2000)

18. Najman, L., Cousty, J.: A graph-based mathematical morphology reader. Pattern Recognition Letters 47, 3-17 (October 2014)

19. Najman, L., Géraud, T.: Discrete set-valued continuity and interpolation. In: Proceedings of the 11th International Symposium on Mathematical Morphology (ISMM). LNCS, vol. 7883, pp. 37-48. Springer, Heidelberg (2013)

20. Ray, N., Acton, S.T.: Inclusion filters: A class of self-dual connected operators. IEEE Transactions on Image Processing 14(11), 1736-1746 (2005)

21. Tustison, N., Avants, B., Siqueira, M., Gee, J.: Topological well-composedness and glamorous glue: A digital gluing algorithm for topologically constrained front propagation. IEEE Transactions on Image Processing 20(6), 1756-1761 (2011)

22. Xu, Y., Géraud, T., Najman, L.: Morphological filtering in shape spaces: Applications using tree-based image representations. In: Proceedings of the International Conference on Pattern Recognition (ICPR). pp. 485-488. IAPR, Tsukuba Science City, Japan (November 2012)

23. Xu, Y., Monasse, P., Géraud, T., Najman, L.: Tree-based morse regions: A topological approach to local feature detection. IEEE Transactions on Image Processing 23(12), 5612-5625 (December 2014) 